\title{
Mathematical Principles for Predicting Reliability Control Parameters of Pipe Armature for Transport Energy Systems
}

\author{
Ivan Kaptsov, Volodimir Kotukh, Nataliy Kaptsova, Yuriy Pakhomov, Katerina Paleyeva
}

\begin{abstract}
When assessing the reliability of transport energy systems, such calculation methods and sources of information about changes in the performance of its elements are required, which would allow predicting behavior, including pipefittings in various operating conditions. The question of the mathematical apparatus application and involvement of already known methods for assessing the quality and efficiency of transport energy systems is of particular importance for the science of reliability. These systems and their structural elements, including pipefittings, cannot be isolated from the influence of the environment and the processes that occur in themselves in the case of residual effects that accelerate wear and reduce their initial characteristics.
\end{abstract}

Index Terms: correlation analysis, mathematical model, reliability, transport energy systems, wear.

\section{INTRODUCTION}

The approach to the design, development of manufacturing technology, and the maintenance and repair of pipefittings is changing significantly due to the high demands on the quality and reliability of structural elements of transport energy systems. Pipe-fittings should be considered as an "aging system", which parameters change as a result of wear of precision sealing pairs, fatigue, and aging of materials, leading to a decrease in the reliability and durability of products. Therefore, the question of applying a mathematical apparatus and attracting already developed methods to allow quality assessment and improvement of the efficiency of pipefittings is of particular importance, when conducting research in the direction of improving its reliability.

\section{STATEMENT OF BASIC MATERIAL\& RESULTS}

Finishing and lapping is the most important technological operation of the process of manufacturing and repairing pipefittings. In accordance with the current standard of Ukraine (DSTU 2960-94), finishing and lapping are considered as a process of improving the reliability of the product, using the functioning of each of them in the

Revised Version Manuscript Received on 10 September, 2019.

Ivan Kaptsov, Department of Exploitation of Natural Gas and Thermal Systems NUUE, Kharkiv, Ukraine.

VolodimirKotukh, Department of Exploitation of Natural Gas and Thermal Systems NUUE, Kharkiv, Ukraine.

NataliyKaptsova, Department of Exploitation of Natural Gas and Thermal Systems NUUE, Kharkiv, Ukraine.

YuriyPakhomov, Department of Exploitation of Natural Gas and Thermal Systems NUUE, Kharkiv, Ukraine.

KaterinaPaleyeva, Department of Exploitation of Natural Gas and Thermal Systems NUUE, Kharkiv, Ukraine. prescribing environmental conditions.

In the implementation of finishing and lapping works with the wrong choice of abrasive mixture, the process of "caricaturing" (pressing in abrasive particles) of the softer surface, being processed, occurs, which is the cause of the technological heredity manifestation. These processes are variations of mechanical wear, caused by the presence of abrasive particles in the parts of conjugate components. During the maintenance of pipe fittings of transport energy systems, there may be cases when foreign solid particles can move through the pipeline at high speed. The presence of abrasive particles in the pipeline with the active chemical action on the metal of the gas environment creates conditions for its rapid wear [1, 2, 3].

Due to the fact, that every detail has a leading type of wear, it will have the most wear surface where this type of wear occurs. A distinct form of wear usually determines the wear resistance of the part, the nature and rate of its wear. Based on this, the concepts of "allowable" and "limit" wear were introduced in the practice of operating transport energy systems. To control the reliability of high-precision products - pipe fittings, we analyze the "allowable" and "limit" wear, considering the wear curve of the most common case of natural mechanical wear as a result of friction (Fig. 1). The horizontal axis shows the time of work of the part in hours, the vertical axis shows the amount of wear.

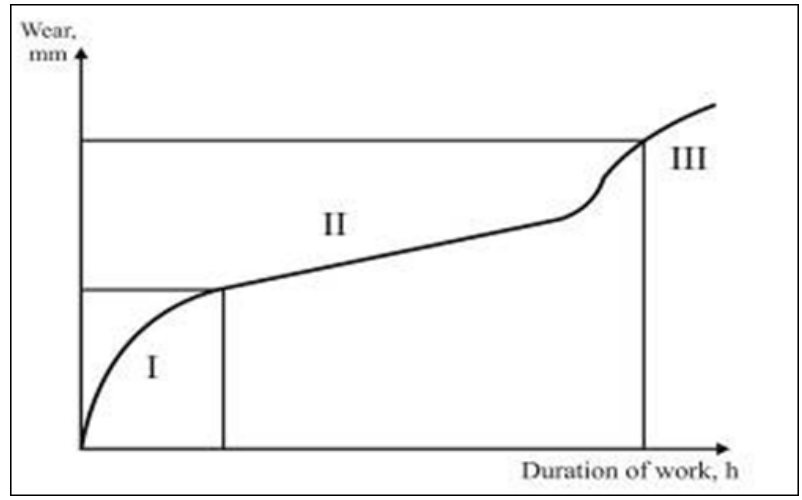

Fig. 1: Typical parts wear curve.

This curve is valid for the majority of conjugate pairs of pipe fit-tings operating in the steady state and it has three particularly pronounced sections: the initial curvilinear section I, which characterizes the process of alignment of the new matching; the straight line section II is the largest in length, corresponding to the period of normal mate operation,

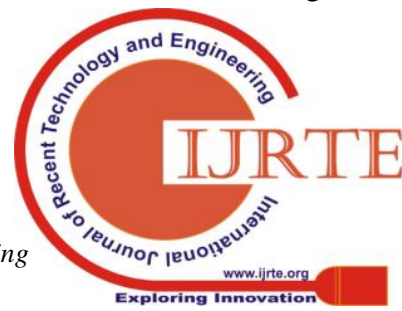


that is, normal wear takes place, and the curved section III, which corresponds to the period of time of matching failure due to wear over its allowable limit (wear limit)

Based on this, it is necessary to consider some mathematical principles for predicting the parameters for controlling the reliability of pipefittings.

The most important task is to distinguish and describe the techno-logical system, investigated for establishing the final properties of the product, depending on its properties at the previous stages. Such a system consists of elements and links between them. The solution of complex problems of ensuring the reliability of pipefittings of transport energy systems largely depends on how the requirements are taken into account and the most important principles of an integrated and systematic approach to its assessment are observed $[4,5]$. When studying the phenomena of technological heredity, a correlation analysis can be used, where the autocorrelation function obtained by means of calculations shows the degree of inheritance of two random variables. With regard to the conditions of reliability properties formation, for example, cork cocks of transport energy systems during polishing, it is possible to determine the influence of the initial roughness profile on the roughness of finishing. Such a problem is solved based on experimental data, using the normalized mutual correlation coupling function $[5,6,7,8]$ :

$$
K_{R_{\mathrm{P}} R_{\mathrm{S}}}(\Delta l)=\frac{M\left[y_{\mathrm{P}}\left(l_{i}\right) y_{\mathrm{S}}\left(l_{i+m}\right)\right]}{\sigma_{\mathrm{P}}\left(l_{i}\right) \sigma_{\mathrm{S}}\left(l_{i}\right)},
$$

where $K_{R_{\mathrm{P}} R_{\mathrm{S}}}(\Delta l)$ is the mutual correlation function of the connection of the polished and lapped profiles;

$M\left[y_{\mathrm{P}}\left(l_{i}\right) y_{\mathrm{S}}\left(l_{i+m}\right)\right]$ is the mathematical expectation of the product of centered profiles;

$y_{\mathrm{P}}\left(l_{i}\right)$ is the current centered value of the coordinate of the profile point of the polished surface on $l_{i}$ segment;

$y_{\mathrm{S}}\left(l_{i+m}\right)$ is the current centered value of the coordinate of the profile point of the smoothed surface on $l_{i+m}$ $(m=0,1,2, \ldots)$ segment;

$\sigma_{\mathrm{P}}\left(l_{i}\right)$ is the standard deviation of the coordinates of the polished profile for any segment of $l_{i}$ surface;

$\sigma_{\mathrm{S}}\left(l_{i}\right)$ is the standard deviation of the coordinates of the smoothed profile for any segment of $l_{i}$ surface.

Analysis of the experimental data showed that the lapped surface profile has a hereditary relationship with genetic profile of the original, in particular, polished surface. By means of the analysis, it was possible to establish, that for the limit conditions of polishing and the subsequent lapped sample in 60 cases out of 100, at the top of the micro-profile of the polished surface there will be the top of the micro-profile of the lapped surface, as well as to establish the effect of roughness on the formation of neighboring roughness $[9,10]$. Correlation analysis is convenient for describing the quantitative aspect of technological heredity of true precision pairs of ball and cork cocks. However, the use of the correlation analysis method requires a large number of actual measurements, which presents significant difficulties, especially for evaluating the high-precision details of pipefittings of transport energy systems under operating

\section{conditions.}

To assess the technical condition and ensure the required reliability of pipe-fittings, a method is proposed whose initial information is passport data on a product, mathematical models and experimental results of characteristics as a function of the amount of wear (defects) of the locking part of pipefittings.

In the practice of operating transport energy systems, the technical condition of pipefittings is usually assessed according to passports (certificates) and actual performance:

- new or repaired pipefittings with defects in the locking part, the magnitude and rate of change of which do not exceed the limits;

- faulty pipefittings with the presence of wear (defects of the locking part, the value of which goes beyond the limits of permissible reliability for tightness);

- in operation of pipefittings that require verification of reliability, where at least one of the estimated criteria of reliability is outside the tolerance limit.

Theoretical analysis of the assessment of reliability for two types of pipefittings characteristics (passport and test) requires the introduction of one more type of characteristics this is the real fact. This characteristic of reliability, determined by the values of specific defects at the time of measuring the parameters of locking fittings, is designed to calculate the reliability and actual presented characteristics of pipefittings. The true actual characteristics are calculated using the same gas-dynamic model as the other given characteristics with zero errors of the channels for measuring the parameters of the pipefittings.

The error in the calculation of the actual presented characteristics is estimated relative to the true actual values from the presented compression ratio and the polytrophic efficiency as a function of the reduced volume throughput of the transport energy systems.

This method is based on the definition of a finite set of generalized defects of the closure part of pipefittings $\left\{D_{j}\right\}$ $j=\overline{1, N D}$ and finding a pair of design characteristics for each element of this set (type of generalized defect): reduced degree of tightness (compression) and polytrophic efficiency as a function of the magnitude of this type of defect and reduced throughput formulas:

$$
\begin{aligned}
& \varepsilon_{\mathrm{r}}=\varepsilon_{\mathrm{r}}^{\mathrm{p}}\left(D_{j}, Q_{\mathrm{r}}\right), \\
& \eta_{\mathrm{p}}=\eta_{\mathrm{p}}^{p}\left(D_{j}, Q_{\mathrm{r}}\right) .
\end{aligned}
$$

For each measurement of $X_{i}$ parameters, based on the calculation of the parameters of the pipefittings, the polytrophic efficiency $\eta_{\mathrm{p}}\left(X_{i}\right)$ and the reduced degree of tightness of the zero approximation $\varepsilon_{\mathrm{r}}^{0}\left(X_{i}\right)$ with $Q_{\mathrm{r}}$ equal to $0,5 \cdot\left(Q_{\min }+Q_{\max }\right)$ are calculated, where $Q_{\min }, Q_{\max }$ are the minimum and maximum values of the range of $Q_{\mathrm{r}}$ variation. Further, it is possible to solve the system of equations (4) $[11,12,5]$ for each type of the generalized 
defect $D_{j}$, on the basis of the calculated characteristics by the method of successive approximations:

$$
\begin{aligned}
& \varepsilon_{\mathrm{r}}^{S}\left(X_{i}\right)=f_{\varepsilon}\left(X_{i}, Q_{\mathrm{r} i, j}^{S}\right), \\
& \varepsilon_{\mathrm{r}}^{S}\left(X_{i}\right)=\varepsilon_{\mathrm{r}}^{p}\left(D_{i, j}^{S}, Q_{\mathrm{r} i, j}^{S}\right), \\
& \eta_{\mathrm{p}}\left(X_{i}\right)=\eta_{\mathrm{p}}^{p}\left(D_{i, j}^{S}, Q_{\mathrm{r} i, j}^{S}\right),
\end{aligned}
$$

where $S$ is the approximation number;

$f_{\varepsilon}$ is the function that determines the dependence of the reduced degree of tightness (accuracy) on the vector of $X_{i}$ changes and reduced throughput (performance).

Solving the system of equations (4) one can find the values $D_{i, j}\left(X_{i}\right)=D_{i, j}^{S}, Q_{\mathrm{r} i, j}\left(X_{i}\right)=Q_{\mathrm{r} i, j}^{S}$, if $\left|D_{i, j}^{S-1}-D_{i, j}^{S}\right|<\delta D$, $\left|Q_{\mathrm{r} i, j}^{S-1}-Q_{\mathrm{r} i, j}^{S}\right|<\delta Q_{\mathrm{r}}$, where $\delta D, \delta Q_{\mathrm{r}}$ are the constants determining the required accuracy of the equations system solution.

As a result of solving this system of equations, an array of values of the generalized defects of pipefittings $\left\{D_{i, j}\left(X_{i}\right)\right\}$ $i=\overline{1, N I}, j=\overline{1, N D}$ is formed.

The following reasons may be obstacles to formula (4) use:

- errors in measuring the reliability parameters of pipefittings by one or more number of evaluation criteria exceed the permissible ones, that is, the transport energy system is in a pre-emergency situation;

- the set of measurement errors of the transported energy carrier at the input and output of any structural element of the transport energy system is such that the calculation of values $\varepsilon_{\mathrm{r}}^{S}\left(X_{i}\right)$ and $\eta_{\mathrm{p}}\left(X_{i}\right)$ lies in the range of values determined by the calculated reduced characteristics;

- the generalized defect, for which the system is being solved, differs essentially from the most common specific defects of the locking part of the pipefittings.

Having calculated the arrays of the values of the generalized defects for the entire series of measurements $\left\{D_{i, j}\left(X_{i}\right)\right\} i=\overline{1, N I}, j=\overline{1, N D}$, the criteria selection of the generalized defect $D_{c}$ from the set $\left\{D_{j}\right\} j=\overline{1, N D}$ is carried out, ensuring maximum certainty of the assessment of the transport energy system reliability.

In the evaluation of the objective compliance measure, characterizing reliability, the deviation function of the calculated value of the pipefittings generalized defect $\left\{D_{i, j}\left(X_{i}\right)\right\}$ from its calculated value $D_{i, j}^{r}$ is adopted. The calculated value of the generalized defect $D_{i, j}^{r}$ is determined based on a statistical analysis of the results of calculating the values of the $j$-th defect type over the entire time interval for measuring the pipefittings performance parameters. Formula dependencies for its definition are well known [12, 5, 9].

Certainty of assessment of the transport energy system and pipefittings reliability is ensured by minimizing the standard deviation calculated by a series of measurements. In this case, the type of the generalized defect $D_{c}$ in pipefittings is selected from condition:

$$
\sum_{i=1}^{N I}\left(D_{i, c}\left(X_{i}\right)-D_{i, c}^{r}\right)^{2}<\sum_{i=1}^{N I}\left(D_{i, \mathrm{j}}\left(X_{i}\right)-D_{i, \mathrm{j}}^{r}\right)^{2},
$$

for $j=1, \ldots, c-1, c+1,111$. ND under the conditions $V D_{\min }<V D_{c}<V D_{\max }$ and $D_{\min }<M\left(D_{c}\right)<D_{\max }$, where $V D_{c}$ is the average accuracy over a series of measurements of the generalized defect change;

$M\left(D_{c}\right)$ is the mathematical expectation of the generalized defect $D_{c}$ for a series of measurements;

$V D_{\min }, V D_{\max }, D_{\min }, D_{\max }$ are the limits restricting changes in the nature and size of the generalized defect in pipefittings for the good product.

In the statistical analysis of arrays $\left\{D_{i, j}\left(X_{i}\right)\right\} i=\overline{1, N I}$, $j=\overline{1, N D}$ the cases are possible, when the estimated average values of the accuracy of change in generalized defects or their values by their small amount, in which the values of standard deviations less than in other types of generalized defects, exceed the permissible limits. In this case, a message is formed about the need to check the reliability of the measurement of pipefittings work reliability parameters or about the need to repair its locking part.

The actual reduced characteristics of pipefittings are estimated based on the calculated reduced characteristics and the calculated value of the selected generalized defect $D_{c}$ :

$$
\begin{aligned}
& \varepsilon_{\mathrm{r}}^{f}\left(Q_{\mathrm{r}}\right)=\varepsilon_{\mathrm{r}}^{p}\left(Q_{\mathrm{r}}, D_{c}\right), \\
& \eta_{\mathrm{p}}^{f}\left(Q_{\mathrm{r}}\right)=\eta_{\mathrm{p}}^{p}\left(Q_{\mathrm{r}}, D_{c}\right) .
\end{aligned}
$$

The third reduced actual characteristic $(N / \rho)_{\mathrm{r}}^{f}\left(Q_{\mathrm{r}}\right)-$ is the reduced internal actual capacity, which is determined using the characteristics $\varepsilon_{\mathrm{r}}^{f}\left(Q_{\mathrm{r}}\right)$ and $\eta_{\mathrm{p}}^{f}\left(Q_{\mathrm{r}}\right)$ already obtained from formulas (6) and (7) using the gas-dynamic model.

When testing for wear and durability, there is a significant variation in the values of the pipe fitting resources of transport energy systems. This variation is caused by the difference in the properties of the metal in different melting or by the use of various technological processes for the manufacture of precision parts, as well as by the difference in the conditions of assembly, testing, operation and repair of the transport energy systems.

The impact of these factors can be significantly reduced by improving the design, improving the technology of manufacturing and assembly, further improving the methods of operation and repair of pipefittings of transport energy systems.

\section{CONCLUSION}

Finishing and lapping are the most important technological operations of the process of manufacturing and repairing pipefittings.

In the implementation of finishing and lapping works with the wrong choice of abrasive mixture, the process of 


\section{Mathematical Principles for Predicting Reliability Control Parameters of Pipe Armature for Transport Energy Systems}

"caricaturing" (pressing in abrasive particles) of the softer surface being processed occurs, this is the reason for the emergence of technological heredity.

Every detail of pipefittings has a leading form of wear, so it will have the most wear surface where this type of wear is manifested.

When studying the phenomena of technological heredity, a correlation analysis can be used, where the autocorrelation function obtained by means of calculations shows the degree of inheritance of two random variables.

Analysis of the experimental data shows that the profile of the lapped surface has a hereditary relationship with the profile of the original ground surface.

Theoretical analysis of the assessment of reliability for two types of pipefittings characteristics (passport and test) requires the introduction of one more type of characteristics, namely, truly actual one.

Certainty of the reliability assessment of the transport energy sys-tem and its structural elements is ensured by minimizing the standard deviation calculated by a series of measurements.

The presence of the obtained reduced actual characteristics of pipefittings makes it possible to assess the technical condition of the transport energy system by the current vector of measurements of its parameters.

\section{REFERENCES}

1. P. P. Borodavkin, Underground trunk pipelines. Moscow: Nedra, 2002.

2. V. Ya. Belyaeva, A. M. Mikhailichenko, A. N. Baraz, R. D. Gabelaya, P. V. Goryunov, Oil and gas construction: study guide. Moscow: Omega-L, 2005.

3. G. V. Konnova, Oil and gas transport and storage equipment. Rostov-on-Don: Phoenix, 2006.

4. V. V. Maslovsky, A. S. Polyansky, Problems of reliability of structural elements of pipeline transport systems, Materials of the International Scientific and Technical Conference. KNAUE, Kharkov (2008), pp. 124-125.

5. I. V. Kuzmin, Fundamentals of modeling complex systems. Kiev: Higher School, 1981.

6. A. V. Alifasov, Technological processes of plastic deformation in mechanical engineering. Minsk: Science and Technology, 1990.

7. P. I. Yascheritsyn, Yu.V. Skorinin, Efficiency of friction units of machines. Minsk: Science and technology, 1994.

8. I. I. Kaptsov, V. G. Kotukh, Yu. V. Pakhomov, Technology of repair of gas equipment and piping systems: monograph. Kharkiv: Kharkiv National University of Urban Economics named after O. M. Beketov, 2016.

9. M. M. Tenenbaum, Wear resistance of structural materials and parts. Moscow: Mechanical Engineering, 1996.

10. V. V. Maslovsky, I. I. Kaptsov, I. V. Sokruto, Fundamentals of technology for the repair of gas equipment and piping systems. Moscow: High School, 2007.

11. B. S. Ilchenko, B. I. Izmalkov, Theoretical foundations and methods for calculating the functional and technical state of gas pumping units: monograph. Kharkov: Collegium, 2006.

12. B. I. Kostetsky, I. G. Nosovsky, Wear resistance and antifriction of machine parts. Kiev: Technika, 1995.

\section{AUTHORS PROFILE}

Ivan Kaptsov - Head of the Department of Exploitation of Natural Gas and Thermal Systems, Kharkiv National University of Urban Economy named after O. M. Beketov, Ukraine, Doctor of Technical Sciences, Professor

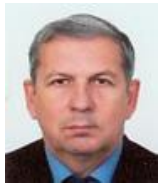

Volodimir Kotukh - Associate Professor of the Department of Exploitation of Natural Gas and Thermal Systems, Kharkiv National University of Urban Economy named after O. M. Beketov, Ukraine, Candidate of Technical Sciences, Docent

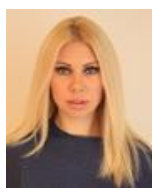

NataliyKaptsova Associate Professor of the Department of Exploitation of Natural Gas and Thermal Systems, Kharkiv National University of Urban Economy named after O. M. Beketov, Ukraine, Candidate of Technical Sciences

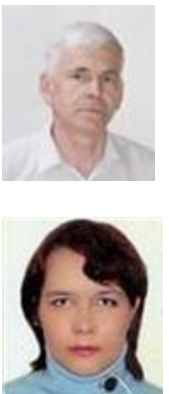

Yuriy PakhomovAssistant of the Department of Exploitation of Natural Gas and Thermal Systems, Kharkiv National University of Urban Economy named after O. M. Beketov, Ukraine

Katerina Paleyeva Assistant of the Department of Exploitation of Natural Gas and Thermal Systems, Kharkiv National University of Urban Economy named after O. M. Beketov, Ukraine 